\title{
RESECTION MARGINS, EXTRAPLEURAL NODAL STATUS, AND CELL TYPE DETERMINE POSTOPERATIVE LONG-TERM SURVIVAL IN TRIMODALITY THERAPY OF MALIGNANT PLEURAL MESOTHELIOMA: RESULTS IN 183 PATIENTS
}

David J. Sugarbaker, MD

Raja M. Flores, MD

Michael T. Jaklitsch, MD

William G. Richards, PhD

Gary M. Strauss, MD

Joseph M. Corson, MD

Malcolm M. DeCamp, Jr, MD

Scott J. Swanson, MD

Raphael Bueno, MD

Jeanne M. Lukanich, MD

Elizabeth Healey Baldini, MD, MPH

Steven J. Mentzer, MD
Objectives: Our aim was to identify prognostic variables for long-term postoperative survival in trimodality management of malignant pleural mesothelioma. Methods: From 1980 to 1997, 183 patients underwent extrapleural pneumonectomy followed by adjuvant chemotherapy and radiotherapy. Results: Forty-three women and 140 men (age range 31-76 years) had a median follow-up of 13 months. The perioperative mortality rate was $3.8 \%$ (7 deaths) and the morbidity, 50\%. Survival in the 176 remaining patients was $38 \%$ at 2 years and $15 \%$ at 5 years (median 19 months). Univariate analysis identified 3 prognostic variables associated with improved survival: epithelial cell type (52\% 2-year survival, $21 \%$ 5-year survival, 26-month median survival; $P=.0001$ ), negative resection margins $(44 \%$ at 2 years, $25 \%$ at 5 years, median 23 months; $P=$ $.02)$, and extrapleural nodes without metastases (42\% at 2 years, $17 \%$ at 5 years, median 21 months; $P=.004)$. Using the Cox proportional hazards, the relative risk of death was calculated for nonepithelial cell type (OR 3.0, CI 2.0-4.5; $P<.0001$ ), positive resection margins (OR 1.7, CI 1.2-2.6; $P=.0082$ ), and metastatic extrapleural nodes (OR 2.0, CI 1.33.2; $P=.0026)$. Thirty-one patients with 3 positive variables had the best survival (68\% 2-year survival, 46\% 5-year survival, median 51 months; $P=.013)$. A previously published staging system using these variables stratified survival $(P<.05)$. Conclusions: $(1)$ Multimodality therapy including extrapleural pneumonectomy is feasible in selected patients with malignant pleural mesotheliomas, (2) pre-resectional evaluation of extrapleural nodes may select patients for radical therapy, (3) microscopic resection margins affect long-term survival, highlighting the need for further investigation of locoregional control, and (4) patients with epithelial, margin-negative, extrapleural node-negative resection had extended survival. (J Thorac Cardiovasc Surg 1999;117:54-65) t was estimated that 2200 to 3000 new cases of mesothelioma would be diagnosed in the United States in 1998. ${ }^{1-3}$ The natural history of malignant pleural mesothelioma includes a median survival of 4 to 12 months without intervention, ${ }^{4-6}$ and there is no accepted standard therapy. The biologic behavior of malignant pleural mesothelioma is unique among thoracic malignant disease. It has shown a natural history of relentless

From the Division of Thoracic Surgery and the Department of Pathology, Brigham and Women's Hospital; Surgical Services and the Department of Adult Oncology, Dana-Farber Cancer Institute, Harvard Medical School, Boston, Mass; and Hematology-Oncology, University of Massachusetts, Worcester, Mass.

Read at the Seventy-eighth Annual Meeting of The American Association for Thoracic Surgery, Boston, Mass, May 3-6, 1998. local progression with rare hematogenous spread even in the late stages of untreated disease. ${ }^{7}$ Even after aggressive local control measures, locoregional recurrence is the fate of a majority of patients. ${ }^{8}$

Extended survival has been reported in patients undergoing multimodality therapy of radical extrapleural pneumonectomy followed by chemotherapy and radiation. ${ }^{9-13}$ The difficulty in this management of the dis-

Received for publication May 8, 1998; revisions requested June 30, 1998; revisions received Sept 25, 1998; accepted for publication Sept 29, 1998

Address for reprints: David J. Sugarbaker, MD, Division of Thoracic Surgery, Brigham and Women's Hospital, Boston, MA 02115.

Copyright (C) 1999 by Mosby, Inc.

$0022-5223 / 99 \$ 8.00+0 \quad \mathbf{1 2 / 6 / 9 4 8 1 9}$ 
ease has been to differentiate patients most likely to benefit from this aggressive approach from those patients destined to die early of the disease.

Since 1980, The Brigham and Women's Hospital applied extrapleural pneumonectomy for malignant pleural mesothelioma in the context of trimodality therapy. Two earlier reports of this series have reviewed the results of 52 and 120 patients. ${ }^{13,14}$ We now review our results with 183 consecutive patients and analyze prognostic variables among the 176 long-term survivors. These variables form the basis of a revision of our published ${ }^{14}$ staging system. We also wish to assess the feasibility of this approach in the light of this larger patient cohort in preparation for future clinical trials and alternative treatment strategies.

\section{Patients and methods}

We reviewed 183 consecutive patients with diffuse malignant pleural mesothelioma treated with extrapleural pneumonectomy followed by adjuvant chemotherapy and radiotherapy from 1980 to 1997 at The Brigham and Women's Hospital, the Dana-Farber Cancer Institute, and the Joint Center for Radiation Therapy (Boston, Mass). Survival data were obtained by reviewing hospital and office records and by contacting patients or their primary care physicians. All surviving patients were cross-sectionally contacted in September 1997.

All pathologic material establishing the diagnosis of malignant pleural mesothelioma was reviewed by The Brigham and Women's Hospital Pathology Department for confirmation. If a definitive diagnosis could not be made on tissue available, a pleuroscopy and biopsy under direct vision was performed. A multimodality team comprising a medical oncologist, a surgeon, and a radiation oncologist evaluated all patients with histologically proven malignant pleural mesothelioma.

Patients were considered surgical candidates if they had a Karnofsky performance status of greater than $70 \%$, a creatinine level within normal limits, liver function test results within the normal range, and tumor judged to be completely resectable on the basis of computed tomographic scan, magnetic resonance imaging (MRI), and echocardiography. Physiologic exclusion criteria included room air arterial $\mathrm{PCO}_{2}$ greater than $45 \mathrm{~mm} \mathrm{Hg}$, room air arterial $\mathrm{PO}_{2}$ less than $65 \mathrm{~mm}$ $\mathrm{Hg}$, echocardiography demonstrating an ejection fraction of less than $45 \%$, and a predicted postoperative forced expiratory volume in 1 second $\left(\mathrm{FEV}_{1}\right)$ of less than $1 \mathrm{~L}$. Patients with a preoperative $\mathrm{FEV}_{1}$ of less than $2 \mathrm{~L}$ underwent quantitative radionuclide ventilation-perfusion scanning to predict postoperative pulmonary function more accurately.

Trimodality treatment consisted of extrapleural pneumonectomy followed by postoperative intravenous chemotherapy and radiotherapy. Extrapleural pneumonectomy entailed resection of the pleura, lung, diaphragm, and pericardium en bloc. ${ }^{15}$ Separate resection of prior open biopsy sites, thoracoscopy incisions, and chest tube tracks included a 1-cm margin of normal tissue.
A standardized pathologic analysis was routinely undertaken for each specimen. First, a gross examination of the specimen was performed by the pathologist to determine any obvious areas of tumor remaining at the resection margins. Then, approximately 20 sections were taken through each specimen to ascertain whether positive microscopic margins remained along the chest wall. The bronchus, pericardium, and diaphragm were carefully examined for microscopic margins. A positive margin identified on the specimen was then used to direct postoperative thoracic radiation treatment.

Extrapleural pneumonectomy at our institution has always included nodal sampling of hilar, paraesophageal, inferior pulmonary ligament, peridiaphragmatic, and subcarinal nodal stations. Additionally, right extrapleural pneumonectomies had sampling of paratracheal nodes, and left extrapleural pneumonectomies included sampling of aortopulmonary window nodes. Involvement of these nodes has been determined in a standardized fashion by the same senior pathologist (J. M. Corson) throughout this series of patients.

Extrapleural nodes are defined as mediastinal lymph nodes and peridiaphragmatic lymph nodes not located within the pleural reflection. Intrapleural nodes are defined as any lymph node located within the pleural envelope. Each patient's status was staged by Butchart criteria, ${ }^{9}$ the new international TNM mesothelioma staging system, ${ }^{16}$ and the system previously published by this group. ${ }^{14}$

Chemotherapy was initiated 4 to 6 weeks after convalescence from extrapleural pneumonectomy. Nine patients treated before 1985 received doxorubicin 50 to $60 \mathrm{mg} / \mathrm{m}^{2}$ and cyclophosphamide $600 \mathrm{mg} / \mathrm{m}^{2}$ for 4 to 6 cycles. Eighty patients treated from 1985 to 1994 also received cisplatin 70 $\mathrm{mg} / \mathrm{m}^{2}$ added to the previous regimen (CAP). Ninety-four patients undergoing treatment from 1995 to 1997 received carboplatin (Paraplatin; Bristol Myers Squibb, Princeton, NJ) and paclitaxel (Taxol; Bristol Myers Squibb) at dosages of an area under the curve (AUC) of 6 and $200 \mathrm{mg} / \mathrm{m}^{2}$, respectively. Although chemotherapy was started between 4 and 6 weeks after extrapleural pneumonectomy, it was started up to 12 weeks after the operation if a patient had major postoperative morbidity. Patients received 2 cycles of carboplatin/ paclitaxel chemotherapy 3 weeks apart, followed by the prescribed course of radiation therapy with concurrent weekly paclitaxel. Beginning 3 to 4 weeks after completion of radiation therapy, patients received 2 additional cycles of carboplatin and paclitaxel 3 weeks apart.

External beam radiotherapy was delivered with the use of linear accelerators ranging in energy from 4 to $15 \mathrm{MV}$. The total radiation dose to the hemithorax was typically $30 \mathrm{~Gy}$ delivered in 1.5-Gy fractions, and the mediastinum received 40 Gy. A boost dose was given to areas of gross residual disease, localized positive resection margins, and/or localized lymph nodes. The boost dose, if given, was typically 14 Gy in 2-Gy fractions for a total cumulative dose to the boost region of $54 \mathrm{~Gy}$.

Because clinical symptoms and radiographic studies are not sensitive enough to accurately diagnose early recurrence, the disease-free interval is difficult to measure. Therefore survival is the major end point of this study. The survival duration was 
measured from the date of extrapleural pneumonectomy until the date of the patient's last follow-up contact or death. Perioperative mortality is defined as death occurring within 30 days of the operation. Morbidity is defined as an untoward event directly resulting in prolonged hospitalization.

Statistical analysis of survival was undertaken by use of a landmark of 30 days after surgical resection. Univariate analysis was performed by means of the Kaplan-Meier lifetable method to determine the effects of demographic and pathologic variables. The log rank test was used to determine statistical significance of comparisons among survival curves. Dichotomous variables included smoking history, asbestos exposure, chest pain, dyspnea, cough, age greater or less than 65 years, side of tumor, sex, cell type (epithelial versus mixed and sarcomatous), tumor at surgical margins, and metastases to extrapleural nodes. Results with a $P$ value of less than .05 were considered significant and were included in a multivariate proportional hazards regression model. This generated an odds ratio of death for each variable associated with shortened survival.

Variables found to be significant on multivariate analysis were used to modify our previously published surgical staging system. Log rank tests were used to determine the statistical significance of survival comparisons between stage groupings.

\section{Results}

Demographics. The overall analysis included 183 patients who initiated therapy by undergoing extrapleural pneumonectomy at The Brigham and Women's Hospital from 1980 to 1997. The cohort included 43 women and 140 men with a mean age of 57 years (range 31-76 years). Forty-nine (27\%) patients were aged 65 years or older. Median follow-up interval was 13 months (range 0.2-100 months) and follow-up is complete through September 1997. Of the 183 patients, 117 (65\%) reported a smoking history and $132(75 \%)$ had known asbestos exposure. One hundred two (56\%) reported a history of chest pain, $133(73 \%)$ reported a history of dyspnea, and 65 (36\%) reported a history of cough. Eighty-two patients (45\%) had pleural mesothelioma of the left side of the chest, and 101 had rightsided tumors.

Morbidity and mortality. Morbidity was divided into major and minor subtypes. Major morbidity was defined as an untoward event leading to longer hospital stay and occurred in 45 of the 183 patients $(24.5 \%)$. Major cardiovascular morbidity occurred in 7 patients: cardiac arrest $(n=5)$, right ventricular failure $(n=1)$, and left ventricular failure $(\mathrm{n}=1)$. Pulmonary morbidity $(\mathrm{n}=15)$ included patients with aspiration $(\mathrm{n}=5)$, pulmonary failure $(\mathrm{n}=5)$, pulmonary embolus $(\mathrm{n}=3)$, and contralateral pneumothorax $(n=2)$. Infectious morbidity $(n=9)$ causes included sepsis $(n=4)$, wound infection $(\mathrm{n}=3)$, empyema $(\mathrm{n}=1)$, and bacteremia ( $=1)$. Gastrointestinal morbidity $(n=7)$ included bleeding in the upper gastrointestinal tract $(\mathrm{n}=2)$, perforated duodenal ulcer $(\mathrm{n}=2)$, colectomy for Clostridium difficile colitis $(\mathrm{n}=1)$, Ogilvie's syndrome $(\mathrm{n}=1)$, and pancreatitis $(n=1)$. Technical morbidity $(n=12)$ included patients who underwent re-exploration for bleeding or suspected cardiac tamponade $(\mathrm{n}=9)$, diaphragmatic patch rupture $(\mathrm{n}=2)$, and cardiac herniation $(\mathrm{n}=1)$. Other miscellaneous morbidity $(\mathrm{n}=18)$ included patients with vocal cord paralysis $(\mathrm{n}=10)$, deep venous thrombosis $(n=4)$, seizure $(n=2)$, and acute renal failure $(\mathrm{n}=2)$.

Minor morbidity included atrial and ventricular arrhythmias, which occurred in 75 patients $(41 \%)$. These arrhythmias were treated with cardioversion or pharmacologic therapy without lengthening hospital stay. Specifically, atrial fibrillation occurred in 68 patients (37\%) and ventricular arrhythmias occurred in 7 patients (3.8\%). Overall, some morbidity occurred in 92 patients $(50 \%)$.

There were 7 perioperative (30-day) deaths (3.8\%). Three patients died of pulmonary embolus, 2 died of myocardial infarction, 1 of cardiac herniation through a pericardial defect, and 1 of respiratory failure. The median postoperative length of stay was 9 days (range 5-101 days).

Long-term survival. The 30-day survival landmark was reached by 176 patients. The median survival for these patients was 19 months; 2- and 5-year survivals were $38 \%$ and $15 \%$, respectively (Fig 1).

Demographic variables not significantly associated with duration of survival within this group of 176 longterm survivors included age greater than or equal to 65 years $(P=.10)$, cigarette use $(P=.33)$, asbestos exposure $(P=.38)$, chest pain $(P=.15)$, dyspnea $(P=.19)$, cough $(P=.92)$, and side of tumor (55\% right; $P=.70)$. Female sex was associated with improved survival on univariate analysis $(P=.03)$ but was not significant in the multivariate model $(P=.16)$.

Epithelial cell type (Fig 2), negative resection margins (Fig 3), and lack of extrapleural lymph nodal involvement (Fig 4) were significant prognostic factors associated with prolonged survival in univariate analysis. The $103(59 \%)$ patients with epithelial cell type tumors had 2- and 5-year survivals of 52\% and 21\%, respectively (Fig 2). By contrast, the survival for the 73 patients with sarcomatous or mixed cell type was $16 \%$ at 2 years, and no patient survived 5 years $(P=.0001$; Fig 2). The 66 patients with negative resection margins had a 2-year survival of $44 \%$ and a 5-year survival of $25 \%$ compared with the 110 patients with positive 


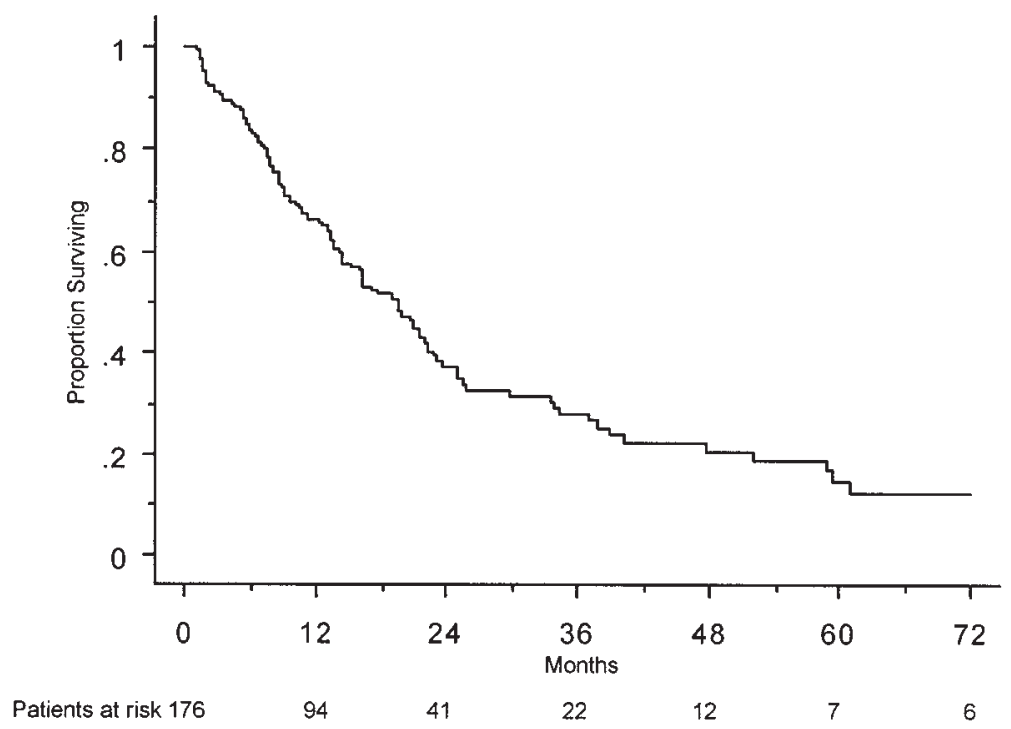

Fig 1. Kaplan-Meier survival curve for all patients surviving surgery $(n=176)$.

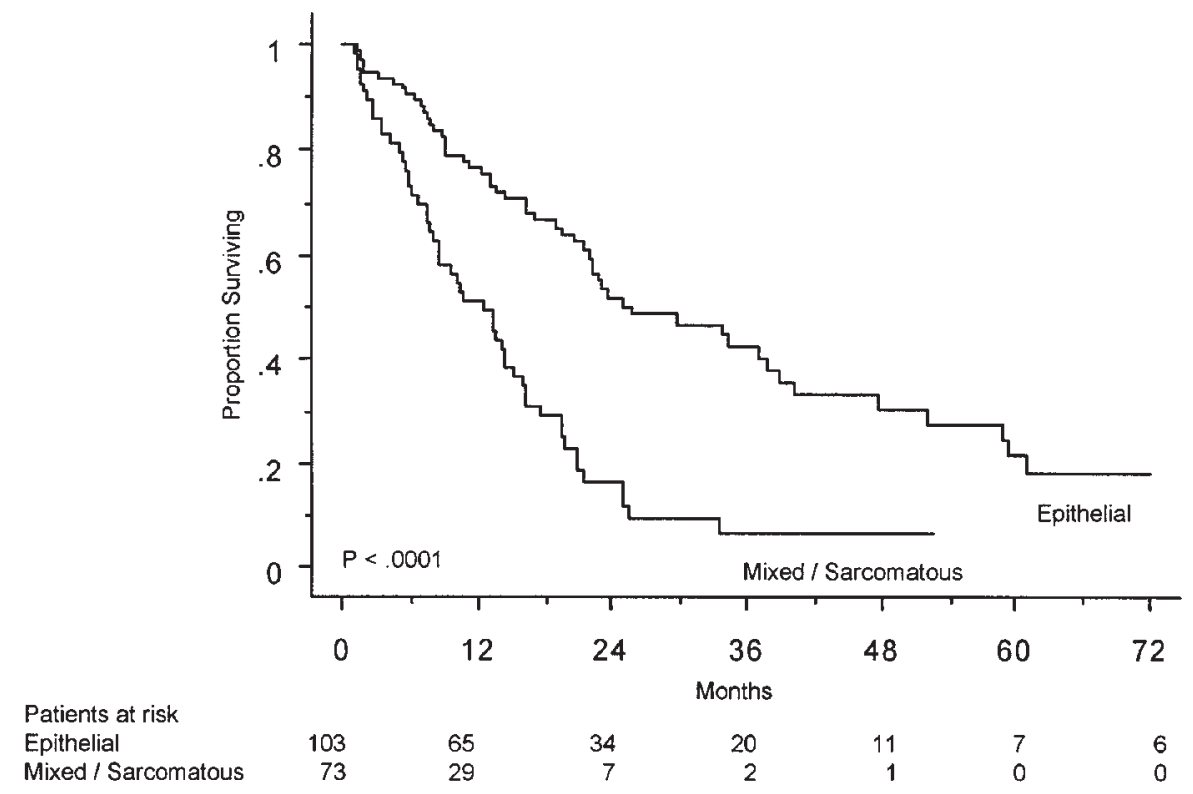

Fig 2. Kaplan-Meier survival curves for patients with epithelial versus sarcomatous and mixed disease $(\mathrm{n}=176)$.

resection margins, who had a 2-year survival of 33\% and a 5 -year survival of $9 \%(P=.02$; Fig 3$)$. The 136 patients with negative extrapleural nodal status had a 2year survival of $42 \%$ and a 5 -year survival of $17 \%$; the 40 patients with positive extrapleural nodal status had a 2 -year survival of 23\%, and none survived 5 years $(P=$ .004 ; Fig 4). Eleven patients had metastases to the extrapleural peridiaphragmatic nodes but not to the mediastinal nodes. We considered metastases to extrapleural peridiaphragmatic nodes to act like metastases to mediastinal nodes because they lay within the same nodal drainage bed as the station 8 (periesophageal) and station 9 (inferior pulmonary ligament) nodes in the American Thoracic Society lymph node map for lung cancer.

The 4 significant variables identified by the log rank test were entered in a Cox proportional hazards model. Gender was no longer a significant predictor of long- 


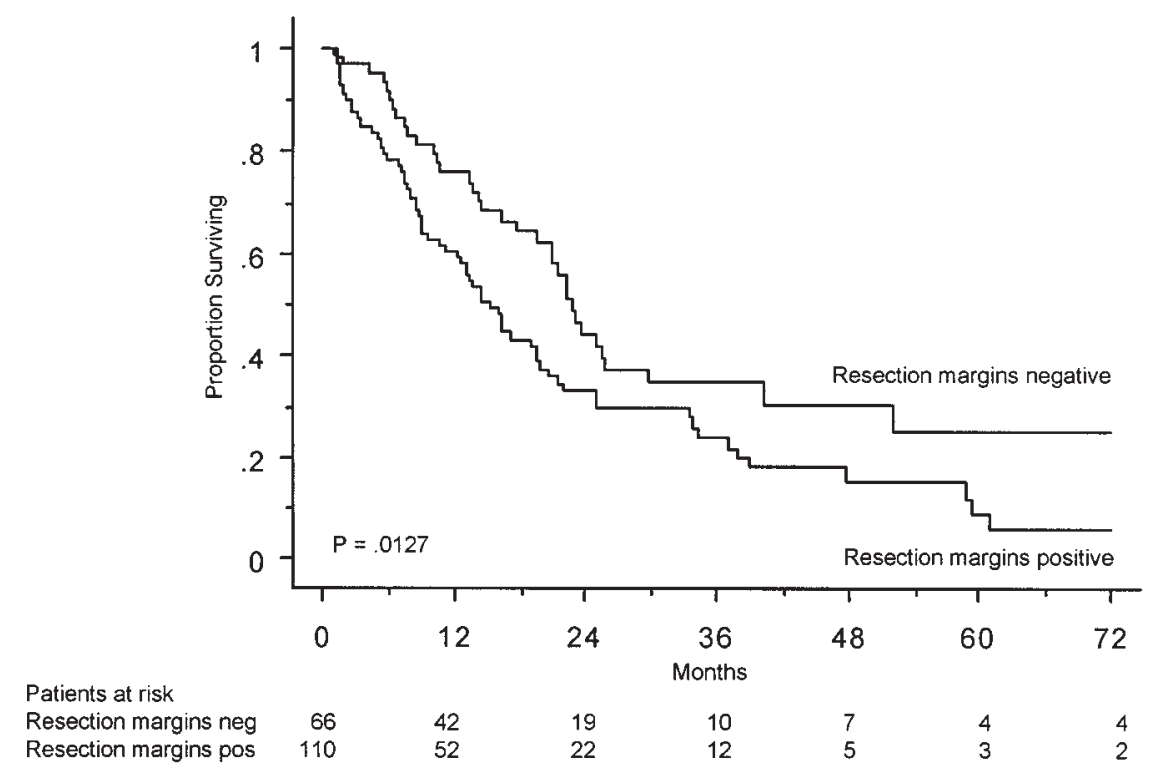

Fig 3. Kaplan-Meier survival curves of patients who had positive versus negative resection margins $(n=176)$.

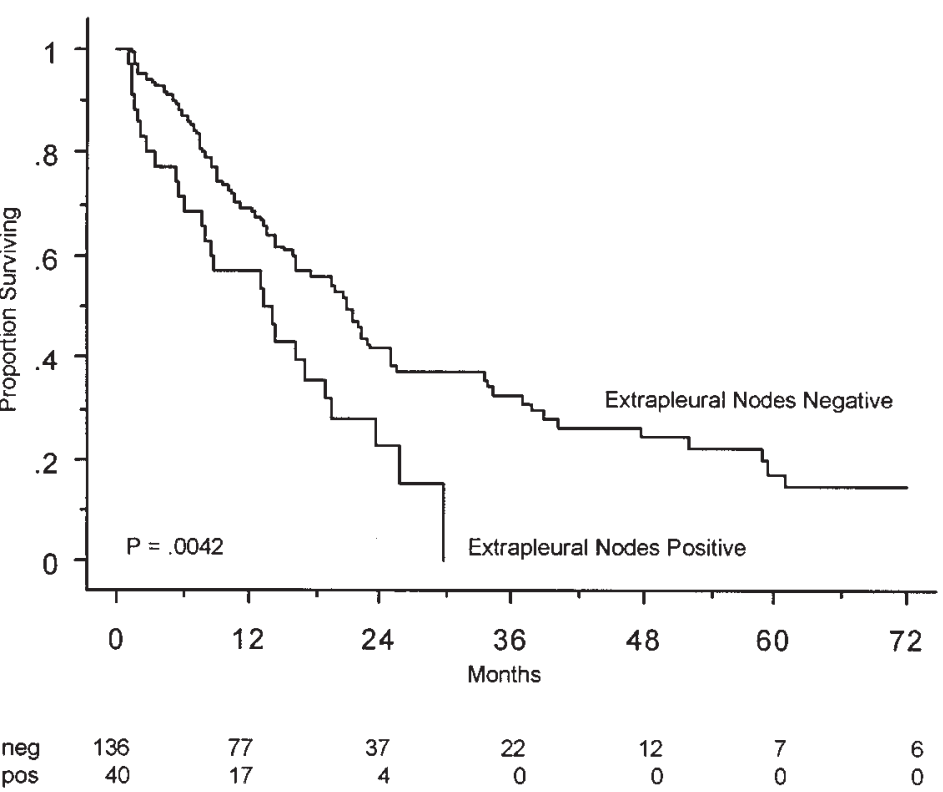

Fig 4. Kaplan-Meier survival curves for patients with positive extrapleural node involvement versus negative extrapleural node involvement $(\mathrm{n}=176)$.

term survival $(P=.03)$. Odds ratios of death and confidence intervals for the remaining 3 prognostic variables are listed in Table I.

Our previously published staging system ${ }^{14}$ was also used to stage this cohort of patients. By this method, survival was significantly stratified by stage $(P=.048)$. Median survival intervals for patients with stage I $(\mathrm{n}=$
66), II $(n=41)$, and III $(n=69)$ disease were 25,20 , and 16 months, respectively. The identification of these predictive variables by the Cox proportional hazards model led us to revise our previous staging system to account for positive margins and extrapleural nodes (Table II). When the criterion of extrapleural nodal involvement was reassigned from stage II to stage III, 


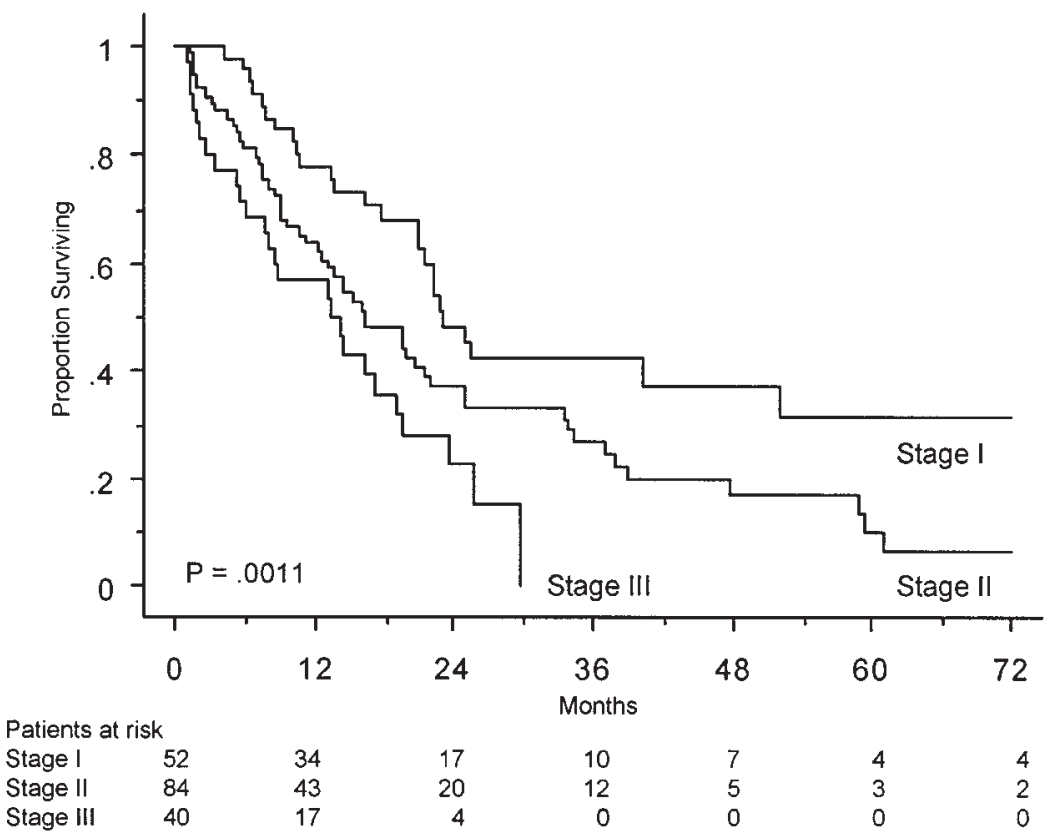

Fig 5. Kaplan-Meier survival curves of all patients surviving surgery: stage $I, n=52$; stage II, $n=84$; and stage III, $\mathrm{n}=40$. Revised staging system $(\mathrm{n}=176 ; P=.0011)$.

Table I. Multivariate outcome of significant prognostic variables $(N=183)$

\begin{tabular}{lrrrr}
\hline Variable & $N$ & OR & CI & P value \\
\hline $\begin{array}{l}\text { Mixed or sarcomatous } \\
\quad \text { cell type }\end{array}$ & 73 & 3.0 & $2.0-4.5$ & $<.0001$ \\
Positive resection margins & 110 & 1.7 & $1.2-2.6$ & .0082 \\
Metastatic extrapleural nodes & 40 & 2.0 & $1.3-3.2$ & .0026
\end{tabular}

$N$, Number of patients; $O R$, odds ratio; $C I$, confidence interval.

survival stratification was improved (Fig 5). This revised staging system significantly stratified long-term survival $(P=.0011)$. This same cohort was not stratified by the new international TNM system for mesothelioma ${ }^{16}(P=.31)$ or by the Butchart staging system ${ }^{9}(P$ $=.09)$.

A subset of 31 patients with epithelial cell type, negative resection margins, and negative extrapleural nodal status had a 51-month median survival with a 2-year survival of $68 \%$ and a 5-year survival of $46 \%$ (Fig 6). This most favorable group with stage I disease and epithelial cell type had significantly better long-term survival than did patients with stage II and stage III epithelial disease $(P=.0044)$.

\section{Discussion}

This study suggests (1) multimodality therapy of malignant pleural mesothelioma combining extrapleur-
Table II. The revised* staging system for malignant pleural mesothelioma

\begin{tabular}{ll}
\hline Stage & \multicolumn{1}{c}{ Description } \\
\hline I & $\begin{array}{c}\text { Disease completely resected within the capsule of the } \\
\text { parietal pleura without adenopathy: ipsilateral pleura, } \\
\text { lung, pericardium, diaphragm, or chest wall disease } \\
\text { limited to previous biopsy sites }\end{array}$ \\
II $\quad \begin{array}{l}\text { All of stage I with positive resection margins and/or } \\
\text { intrapleural adenopathy }\end{array}$ \\
III $\quad \begin{array}{l}\text { Local extension of disease into the chest wall or } \\
\text { mediastinum; heart, or through diaphragm, peritoneum; } \\
\text { or with extrapleural lymph node involvement }\end{array}$ \\
IV $\quad$ Distant metastatic disease
\end{tabular}

Note: Patients with Butchart stage II and III $^{9}$ disease are combined into stage III. Stage I represents patients with resectable disease and negative nodes. Stage II indicates resectable disease but positive nodes.

*Revised from Sugarbaker DJ, Strauss GM, Lynch TJ, Richards W, Mentzer SJ, Lee TH, et al. Node Status Has Prognostic Significance in the Multimodality Therapy of Diffuse, Malignant Mesothelioma. J Clin Oncol 1993;11:1172-8; published with permission of WB Saunders Company).

al pneumonectomy with adjuvant chemoradiotherapy is feasible with acceptable perioperative mortality (3.8\%); (2) a subgroup of patients with epithelial cell type, negative extrapleural nodes, and complete resection margins have a long-term median survival approaching 5 years; (3) research efforts should be directed toward devising improved methods of obtaining local control; (4) pre-resectional extrapleural node 


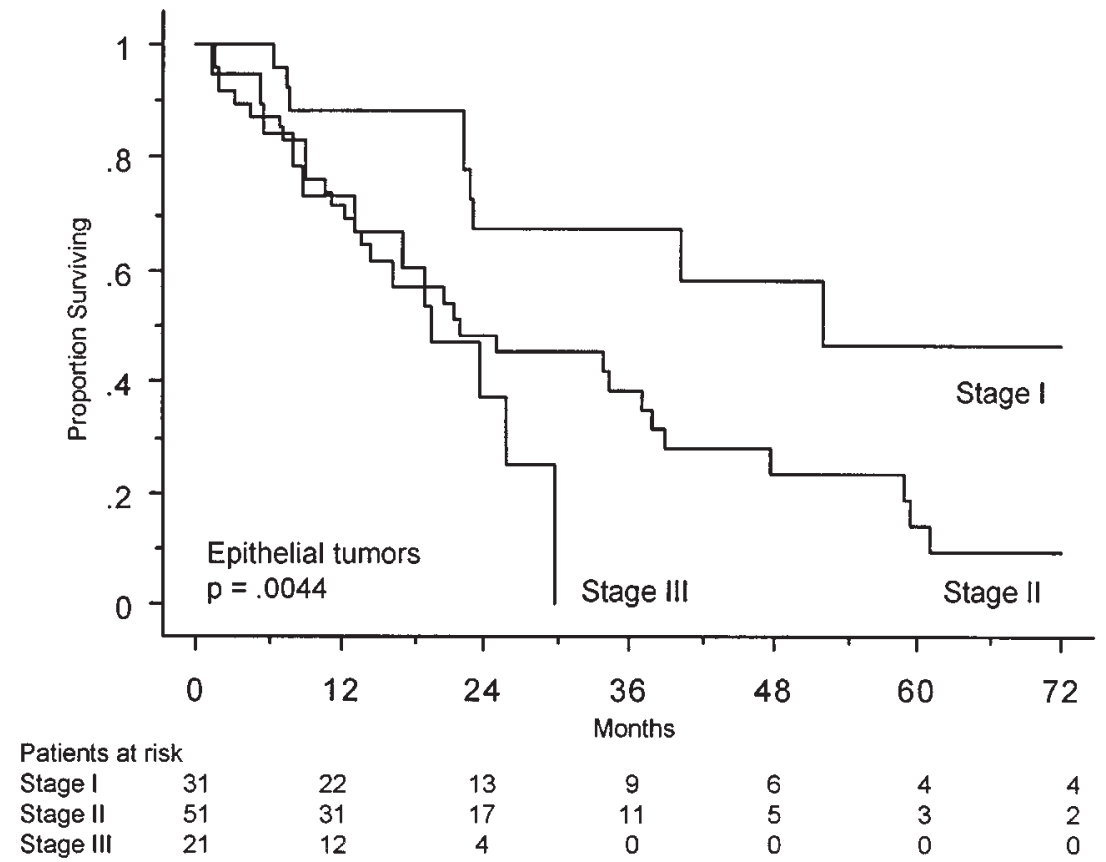

Fig 6. Kaplan-Meier survival curves of patients with epithelial-type tumor: stage I, $\mathrm{n}=31$; stage II, $\mathrm{n}=51$; and stage III, $\mathrm{n}=21(\mathrm{n}=103 ; P=.0044)$.

staging may play an important role in deciding treatment strategy; and (5) the staging system previously published ${ }^{14}$ significantly stratified survival. Finally, we propose a revision to our previously published staging system.

This study demonstrates in a large patient cohort that extrapleural pneumonectomy is a safe means of cytoreduction. In 1976, Butchart and associates ${ }^{9}$ reported their experience with extrapleural pneumonectomy but demonstrated a prohibitively high perioperative mortality rate of $31 \%$. The advent of improved surgical techniques, hemostasis, prosthetic reconstruction of the diaphragm and pericardium, advances in intraoperative and postoperative monitoring, and better critical care support have since improved postoperative outcome to our current rate of 7 deaths in 183 operations (3.8\% mortality).

The major operative morbidity in this cohort was $24.5 \%$, but many of the complications do not present major setbacks when treated appropriately. Atrial fibrillation occurred in $37 \%$ but was well controlled with calcium channel blockers, digoxin, procainamide, or a combination of these agents. No patient had severe consequences as a result of this complication. It is possible that rapid filling within the denuded hemithorax results in respiratory distress because the dense fluid shifts the mediastinum toward the remaining lung and compresses it. Aspiration of the fluid-filled hemithorax, as demonstrated in 6 of our patients, leads to repositioning of the mediastinum, decrease in intra-abdominal pressure, and subsequent lung re-expansion followed by resolution of hypoxia. The attendant morbidity and potential mortality from extrapleural pneumonectomy stresses the importance of performing the procedure at specialized institutions.

The current regimen of combined carboplatin-paclitaxel adjuvant chemotherapy was designed in 1995 . Platinum-based chemotherapy yielded a response in 7 of $15(47 \%)$ patients with peritoneal mesothelioma ${ }^{17}$ and in $18 \%$ with pleural mesothelioma. ${ }^{18}$ Paclitaxel as a single agent in advanced pleural mesothelioma produced regression in 2 of $15(13 \%)$ and stable disease in 5 of $15(33 \%)$ patients treated by the Cancer and Leukemia Group B (CALGB) in 1993. ${ }^{19}$ In 1995 Herscher and colleagues ${ }^{20}$ reported local control of advanced pleural mesothelioma with single-agent paclitaxel in 7 of $8(88 \%)$ patients. Although there were no combination data of carboplatin-paclitaxel in malignant pleural mesothelioma, these 2 agents had recognized single-agent activity against this chemotherapyresistant disease. Furthermore, there were published data on the use of carboplatin-paclitaxel combinations in the treatment of advanced breast, ovarian, and lung cancers with high response rates: $78 \%$ to $94 \%$ in breast 
cancer, ${ }^{21,22} 57 \%$ to $81 \%$ in ovarian cancer, ${ }^{23,24}$ and $38 \%$ to $86 \%$ in lung cancer. ${ }^{25-31}$ The rationale for this approach has been steadily evolving in the literature. Cisplatin and paclitaxel as single agents were each effective against some in vivo mesothelioma cell lines in athymic nude mice, but they were more effective in all cell lines when given in combination. ${ }^{32}$

Carboplatin-paclitaxel was recognized as a better tolerated chemotherapy regimen than the CAP (cyclophosphamide-doxorubicin-platinum) regimen it replaced. Less toxicity for patients after pneumonectomy would suggest more patients will receive adjuvant therapy.

In other types of malignant disease, chemotherapy is more effective in the setting of minimal residual tumor burden, ${ }^{33}$ although this remains unproven in pleural mesothelioma.

Extrapleural pneumonectomy combined with adjuvant chemoradiation therapy by this protocol offers improved survival for certain subgroups of patients. Specifically, patients with epithelial cell type, lack of extrapleural nodal involvement, and negative surgical margins have a median survival approaching 5 years. The patients with non-epithelial cell type (sarcomatoid and mixed cell type) have a significantly worse survival, with only $16 \%$ living for 2 years after the operation. This suggests that our current trimodality treatment plan is having a small impact within this group with unfavorable histologic features, and new strategies for local control are needed. One possibility would be to apply chemotherapy combinations currently administered in the treatment of sarcomas such as MAID (methotrexate, Adriamycin [Pharmacia \& Upjohn, Kalamazoo, Mich], ifosfamide, and prednisone) instead of carboplatin-paclitaxel combinations that have demonstrated responses against epithelial tumors.

Mesothelioma, unlike lung cancer, tends to progress locally rather than systemically. Of the $54 \%$ of patients with recurrences reported by Baldini and colleagues, ${ }^{8}$ $67 \%$ had recurrences within the ipsilateral hemithorax and $50 \%$ had recurrences within abdominal regions; a majority of these recurrences appeared to result from direct extension from the ipsilateral hemithorax. Recurrences are thought to result from trauma, spillage, and residual tumor at the resection margins that subsequently are entrapped in fibrin deposits and become exposed to postoperative growth factors. ${ }^{34}$ In this study, patients with positive resection margins had a median survival of 15 months (33\% 2-year survival, 9\% 5-year survival) and patients with negative resection margins had a median survival of 23 months (44\% 2-year survival, 25\% 5-year survival; $P=.02$ ). Our previous report on the first 120 patients did not demonstrate this finding, ${ }^{13}$ which we attribute to the larger number of patients in the current study cohort. Taken together, these observations suggest that future investigative efforts should be directed toward more effective methods of locoregional control.

Pass and colleagues ${ }^{35}$ have studied the role of intraoperative photodynamic therapy as a method of local control in the treatment of malignant pleural mesothelioma. They have performed a phase I trial to determine the maximally tolerated dose. However, they recently published the results of a phase III trial indicating that this treatment did not appear to prolong survival or improve local control when performed after surgical debulking. ${ }^{36}$

Rusch and colleagues ${ }^{37,38}$ have extensively studied pleurectomy and postoperative intrapleural chemotherapy. Their results have shown only marginal improvement in outcome without significant toxicity. In patients undergoing pleurectomy, gross residual tumor frequently remains within the hemithorax. Given the millimeter level of penetration of intracavitary chemotherapy, the amount of cytoreduction with pleurectomy may be inadequate to allow the intracavitary chemotherapy to work optimally. ${ }^{39-42}$ In patients undergoing extrapleural pneumonectomy, residual tumor within the hemithorax is most often microscopic, presenting a smaller tumor burden to be controlled with intracavitary chemotherapy. Kodama and colleagues ${ }^{43}$ have used hyperthermic intrathoracic chemotherapy in patients undergoing resection for adenocarcinoma with evidence of improved local control. No data exist in patients with malignant pleural mesothelioma after surgical resection.

The dismal survival of patients with extrapleural nodal involvement suggests a role for pre-resectional lymph node staging. Either mediastinoscopy or positron emission tomography (PET) scanning may play a useful role in patient selection. Although paraesophageal lymph nodes are inaccessible by mediastinoscopy, a positive finding of a diseased mediastinal node is useful nonetheless. Data on the detection of mediastinal nodes via mediastinoscopy and PET scanning are nonexistent in patients with mesothelioma. The pattern of mesothelioma tumor spread does not appear to follow an ordered lymphatic pattern like that seen in lung cancer, and studies establishing the sensitivity and specificity of mediastinoscopy in this particular disease are needed.

We recommend that patients with a histologic diagnosis of sarcomatoid or biphasic mesothelioma and comorbid disease undergo mediastinoscopy because the combination of both sarcomatoid or mixed cell 
types and positive extrapleural nodal status predicts a survival that mimics the natural history without surgical intervention. If pre-resectional nodal testing proves positive in high-risk patients with sarcomatoid or mixed tumors, we would not recommend proceeding with extended resection.

The proposed International TNM Staging System ${ }^{16}$ and the Butchart staging system ${ }^{9}$ failed to stratify survival when applied to our cohort of 176 patients. The TNM staging system placed $8 \%$ of our cohort into the stage I category, $11 \%$ into stage II, $78 \%$ into stage III, and $3 \%$ into stage IV. Since the majority of our cohort of 176 patients were categorized as stage III by TNM, it is difficult to separate patients with different tumor characteristics that are necessary to stratify survival in our patient cohort. In addition, the $\mathrm{T}$ descriptor alone was not a statistically significant predictor of survival, reflecting the inability of this system to describe the biologic behavior of this particular tumor.

The system proposed by Butchart similarly did not significantly stratify survival. A small number of patients were categorized as having stage III disease ( $\mathrm{n}$ $=5,3 \%)$. The distinction between extent of primary tumor involvement and intrathoracic nodal involvement is not appropriately taken into account by this staging system, as reflected by the majority of patients being placed in the stage II category.

The staging system proposed previously by this group ${ }^{14}$ continued to stratify survival successfully. This is a surgical staging system based on the ability to completely remove all tumor within the pleural envelope and involved regional nodes. Observer bias may exist because this staging system originated at our institution and was based on an earlier cohort. Validation by other institutions will be required to judge the utility of this clinical staging system.

We considered peridiaphragmatic nodes to be extrapleural nodes in this analysis. Metastasis to peridiaphragmatic nodes was associated with a similar median survival as metastasis to mediastinal nodes. However, if one considers only the 29 patients with metastases to the mediastinal nodes that could be reached by mediastinoscopy, there was a highly significant difference in long-term survival of these patients compared with patients with uninvolved mediastinal nodes $(P=.0026)$.

Because of the negative effect of extrapleural nodal disease on survival, we propose a revised staging system to predict long-term survival (Table II). This staging system differs from our previous publication ${ }^{14}$ in that the presence of extrapleural node involvement has now been included in stage III. This represents a dis- tinction as to the location and extent of nodal involvement based on the poorer survival of patients in this subgroup.

A median survival of 51 months in the subgroup of patients with negative extrapleural lymph nodes is a ray of hope in the treatment of this dismal disease. We believe it is no longer appropriate to offer only supportive care to all patients with mesothelioma because a subgroup of well-selected patients appears to benefit from aggressive multimodality treatment (Fig 6).

We thank John Orav, PhD, for his work on the statistics for this paper and Mary Sullivan Visciano for editorial assistance.

\section{REFERENCES}

1. Spirtas R, Beebe GW, Connelly RR, Wright WE, Peters JM, Sherwin RP, et al. Recent trends in mesothelioma incidence in the United States. Am J Ind Med 1986;9:397-407.

2. Connelly RR, Spirtas R, Myers MH, Percy CL, Fraumeni JF Jr. Demographic patterns for mesothelioma in the United States. J Natl Cancer Inst 1987;78:1053-60.

3. Enterline PE, Henderson VL. Geographic patterns of pleural mesothelioma deaths in the United States, 1968-81. J Natl Cancer Inst 1987;79:31-7.

4. Chahinian AP, Ambinder RM, Mandel EM, Holland JF, Evaluation of 63 patients with diffuse malignant mesothelioma [abstract]. Proc Am Soc Clin Oncol 1980;21:360.

5. Law MR, Hodson ME, Turner-Warwick M. Malignant mesothelioma of the pleura: clinical aspects and symptomatic treatment [abstract]. Eur J Respir Dis 1984;65:162-8.

6. Ruffie P, Feld R, Minkin S, et al. Diffuse malignant mesothelioma of the pleura in Ontario and Quebec: a retrospective study of 332 patients. J Clin Oncol 1989;7:1157-68.

7. Antman KH. Clinical presentation and natural history of benign and malignant mesothelioma. Semin Oncol 1981;8:313-20.

8. Baldini EH, Recht A, Strauss GM, DeCamp MM Jr, Swanson SJ, Liptay MJ, et al. Patterns of failure after trimodality therapy for malignant pleural mesothelioma. Ann Thorac Surg 1997;63:3348.

9. Butchart EG, Ashcroft T, Barnsley WC, Holden MP. Pleuropneumonectomy in the management of diffuse malignant mesothelioma of the pleura: experience with 29 patients. Thorax 1976;31:15-24.

10. Antman K, Pass HI, Recht A. Benign and malignant mesothelioma. In: DeVita VT Jr, Hellman S, Rosenberg SA, editors. Cancer: principles and practice of oncology. 3rd ed. Philadelphia: JB Lippincott; 1989. p. 1399-414.

11. Antman KH, Pass HI, DeLaney T, Li FP, Corson J. Benign and malignant mesothelioma. In: DeVita VT Jr, Hellman S, Rosenberg SA, editors. Cancer: principles and practice of oncology. 4th ed. Philadelphia: JB Lippincott; 1993. p. 1489-508.

12. Sugarbaker DJ, Mentzer SJ, DeCamp M, Lynch TJ Jr, Strauss GM. Extrapleural pneumonectomy in the setting of a multimodality approach to malignant mesothelioma. Chest 1993;103 (suppl):377S-81S.

13. Sugarbaker DJ, Garcia JP, Richards WG, Harpole DH Jr, Baldini HE, DeCamp MM Jr, et al. Extrapleural pneumonectomy in the multimodality therapy of malignant pleural mesothelioma: 
results in 120 consecutive patients. Ann Surg 1996;224:288-94; discussion.

14. Sugarbaker DJ, Strauss GM, Lynch TJ, Richards W, Mentzer SJ, Lee TH, et al. Node status has prognostic significance in the multimodality therapy of diffuse, malignant mesothelioma. J Clin Oncol 1993;11:1172-8.

15. Sugarbaker DJ, Mentzer SJ, Strauss G. Extrapleural pneumonectomy in the treatment of malignant pleural mesothelioma. Ann Thorac Surg 1992;54:941-6.

16. Rusch VW. A proposed new international TNM staging system for malignant pleural mesothelioma. From the International Mesothelioma Interest Group [see comments]. Chest 1995;108: $1122-8$.

17. Markman M, Kelsen D. Efficacy of cisplatin-based intraperitoneal chemotherapy as treatment of malignant peritoneal mesothelioma. J Cancer Res Clin Oncol 1992;118:547-50.

18. Krarup-Hansen A, Hansen HH. Chemotherapy in malignant mesothelioma: a review [abstract]. Cancer Chemother Pharmacol 1991;28:319-30.

19. Vogelzang NJ, Herndon J, Clamon GH, Mauer AM, Cooper MR, Green MR. Paclitaxel (Taxol) for malignant mesothelioma: a phase II study of the Cancer and Leukemia Group B (CALGB 9234) [abstract]. Prog Proc Am Soc Clin Oncol 1994;13:405: 1382.

20. Herscher L, Hahn S, Pass H, Temeck B, Goldspiel B, Cook J, et al. A phase I study of paclitaxel as a radiation sensitizer for the treatment of non-small cell lung cancer and mesothelioma [abstract]. Prog Proc Am Soc Clin Oncol 1995;14:A1530.

21. Tolcher AW, Gelmon KA. Interim results of a phase I/II study of biweekly paclitaxel and cisplatin in patients with metastatic breast cancer. Semin Oncol 1995;22(4 Suppl 8):28-32.

22. Gelmon KA. Biweekly paclitaxel (Taxol) and cisplatin in breast and ovarian cancer. Semin Oncol 1994;21(5 Suppl 8):24-8.

23. Meerpohl HG, duBois A, Kuhnle H, Luck HJ, Kreienberg R, Mobus V, et al. Paclitaxel combined with carboplatin in the firstline treatment of advanced ovarian cancer. Semin Oncol 1995; 22(6 Suppl 15):712.

24. Blois G. Pilot study with fixed-dose carboplatin and escalating paclitaxel in advanced ovarian cancer. Semin Oncol 1995;22(6 suppl 14):32-4

25. Belli L, LeChevalier T, Gottfried M, Adams D, Ruffie P, LeCesne A, et al. Phase I/II study of paclitaxel plus cisplatin as first-line chemotherapy for advanced non-small cell lung cancer: preliminary results. Semin Oncol 1995;22(6 Suppl 15):29-33.

26. Greco FA, Stroup SL, Hainsworth JD. Paclitaxel by 1-hour infusion in combination chemotherapy of stage III non-small cell lung cancer. Semin Oncol 1995;22(4 Suppl 9):75-7.

27. Muggia FM, Vafai D, Natale R, Israel V, Zaretsky S, McRae AM, et al. Paclitaxel 3-hour infusion given alone and combined with preliminary results of dose-escalation trials. Semin Oncol 1995;22(4 Suppl 9):63-6.

28. Strauss GM, Lynch TJ, Elias AD, Jacobs C, Kwiatkowski DJ, Shulman LN, et al. A phase I study of ifosfamide/carboplatin/ etoposide/paclitaxel in advanced lung cancer. Semin Oncol 1995; 22(4 Suppl 9):70-4.

29. Bunn PA Jr, Kelly K. A phase I study of carboplatin and paclitaxel in non-small cell lung cancer: a University of Colorado Cancer Center study. Semin Oncol 1995;22(4 Suppl 9):26.

30. Antonia SJ, Wagner H, Williams C, Alberts M, Hubbell D, Robinson L, et al. Concurrent paclitaxel/cisplatin with thoracic radiation in patients with stage IIIA/B non-small cell carcinoma of the lung. Semin Oncol 1995;22(4 Suppl 9):34-7.

31. Langer CJ, Leighton JC, Comis RL, O'Dwyer PJ, McAleer CA, Bonjo CA, et al. Paclitaxel by 24- or 1-hour infusion in combination with carboplatin in advanced non-small cell lung cancer: the Fox Chase Cancer Center experience. Semin Oncol 1995; 22(4 Suppl 9):18-29.

32. Chahinian AP, Mandeli JP, Gluck H, Naim H, Teirstein AS, Holland JF. Effectiveness of cisplatin, paclitaxel, and suramin against human malignant mesothelioma xenografts in athymic nude mice. J Surg Oncol 1998;67:104-11.

33. Griffiths CT, Grogan RH, Hall TC. Advanced ovarian cancer: primary treatment with surgery, radiotherapy, and chemotherapy. Cancer 1972; 29:1-7.

34. Sugarbaker PH. Observations concerning cancer spread within the peritoneal cavity and concepts supporting an ordered pathophysiology. In: Sugarbaker PH, editor. Peritoneal carcinomatosis: principles of management. Boston: Kluwer Academic Publishers; 1996. p. $79-100$.

35. Pass HI, DeLaney TF, Tochner Z, Smith PE, Temeck BK, Pogrebniak HW, et al. Intrapleural photodynamic therapy: results of a Phase I trial. Ann Surg Oncol 1994;1:28-37.

36. Pass HI, Temeck BK, Kranda K, Thomas G, Russo A, Smith P, et al. Phase III randomized trial of surgery with or without intraoperative photodynamic therapy and postoperative immunochemotherapy for malignant pleural mesothelioma. Ann Surg Oncol 1997;4:628-33.

37. Rusch V, Saltz L, Venkatraman E, Ginsberg R, McCormack P, Burt M, et al. A phase II trial of pleurectomy/decortication followed by intrapleural and systemic chemotherapy for malignant pleural mesothelioma. J Clin Oncol 1994:12:1156-63.

38. Rusch VW. Pleurectomy/decortication in the setting of multimodality treatment for diffuse malignant pleural mesothelioma. Semin Thorac Cardiovasc Surg 1997;9:367-72.

39. Los G, vanVugt MJ, Pinedo HM. Potentiation of Ip chemotherapy by abdominal hyperthermia in rats [abstract]. Proc Ann Meet Am Assoc Cancer Res 1992:33:A2980.

40. Los G, Verdegaal EM, Mutsaers PH, McVie JG. Penetration of carboplatin and cisplatin into rat peritoneal tumor nodules after intraperitoneal chemotherapy. Cancer Chemother Pharmacol 1991;28:159-65.

41. Los G, McVie JG. Experimental and clinical status of intraperitoneal chemotherapy. Eur J Cancer 1990;26:775-62.

42. Jacquet P, Stephens AD, Averbach AM, Chang D, Ettinghausen SE, Dalton RR, et al. Analysis of morbidity and mortality in 60 patients with peritoneal carcinomatosis treated by cytoreductive surgery and heated intraoperative intraperitoneal chemotherapy. Cancer 1996;77:2622-9.

43. Kodama K, Doi O, Tatsuta M, Kuriyama K, Tateishi R. Development of postoperative intrathoracic chemo-thermotherapy for lung cancer with objective of improving local cure. Cancer 1989;64:1422-8

\section{Discussion}

Dr Larry R. Kaiser (Philadelphia, $P a$ ). This series represents the largest series of extrapleural pneumonectomy to date. We have seen data from some of these patients in earlier studies but now have the advantage of looking at the mature data from 183 patients with a median follow-up inter- 
val of 13 months. Amazingly, the overall operative mortality is a phenomenal $3.8 \%$. This is truly impressive.

The data further support the functional staging classification originally proposed by Dr Sugarbaker and his colleagues and now further revised and updated, which further adds to the risk stratification and predictive value of this classification.

I would like to expand on what Dr Sugarbaker has already stated. Using his revised staging criteria, we can make betterinformed decisions regarding appropriate treatment for individual patients. Not all patients with mesothelioma are in a hopeless condition. A significant percentage of patients deserve an aggressive multimodality approach, as the Brigham group has delineated.

Dr Sugarbaker, to what do you attribute the absence of adult respiratory distress syndrome (ARDS) in this large group of patients undergoing extrapleural pneumonectomy? Are you doing something we all should be doing?

Obviously, patient selection is important. To what percentage of patients with malignant pleural mesothelioma do you offer this multimodality approach?

Do you have an arbitrary age cutoff, or is this individualized? Are you performing mediastinoscopy routinely before resection?

This is an aggressive regimen. Do you have any quality-oflife data from the first year of treatment? What percentage of patients have grade IV toxicity during the postoperative chemotherapy-radiotherapy regimen?

Did you find MRI predictive of which patients should be able to have at least a microscopically complete resection, if not a complete resection? Can you base this on MRI criteria?

Dr Sugarbaker. Thank you for your comments. I would like to start by addressing the question of ARDS. We did have some cases of ARDS, which contributed to the $25 \%$ morbidity. ARDS is seen in the same percentage that one would expect in patients undergoing pneumonectomy for non-small cell lung cancer.

However, we have selected these patients via MRI, looking for resectable disease, and by echocardiogram, and we do restrict intravenous fluids, probably not much different from our standard pneumonectomy patients. Nevertheless, I wonder what effect the screening for myocardial function would have on patients undergoing pneumonectomy in general. It may be helping, and it is an unseen benefit.

We do not have an age cutoff, but most of the patients whom we consider for this aggressive strategy are in their fifth or sixth decade. Surprisingly, we are seeing an increasing number of patients in their 40s and 50s with the disease. That does make one reconsider possible new causes, such as the simian 40 virus, but we do not have any specific age cutoff.

We seriously consider using this therapy in about one third of patients whom we see, so we see 3 or 4 for every single patient whom we consider for extrapleural pneumonectomy followed by trimodality therapy.

In terms of mediastinoscopy, PET scanning, or laparoscopy to get at retro-aortic or retro-paraesophageal nodes, these are areas that need to be studied. Dr Rusch published a study on the use of laparoscopy to detect transdiaphragmatic involve- ment or intraperitoneal involvement, but I think we need to move forward with an evaluation of the ability of mediastinoscopy to detect nodal disease in these patients.

Notice that we very carefully suggested that these were extrapleural nodes, and they are really a "grab bag" of mediastinal nodes, and that it is not clear how many of them would be accessible via mediastinoscopy. Nevertheless, I believe that PET will be an interesting tool for pre-resectional staging.

Patients with sarcomatous disease who have positive mediastinal nodes on mediastinoscopy should not be considered for aggressive therapy. Their survival is dismal despite an aggressive approach and the resectability rate is low.

We do not have data on the quality of life in these individuals, but it is an important point and should be studied.

I do not have data on grade IV toxicity at hand. The reason we switched from the CAP protocol (cytoxan, Adriamycin, and then platinol), which was the most active combination chemotherapy against mesothelioma, to carboplatin and paclitaxel was precisely because of the incidence of grade IV toxicity that prevented patients from completing the adjuvant regimen.

We consider MRI to be a better pre-resectional staging modality than computed tomographic scanning, and we believe this has contributed significantly to the resectability rate in our patients, which now approaches $80 \%$. It is indeed superior for looking at mediastinal invasion or transdiaphragmatic invasion.

Dr G. Alexander Patterson (St Louis, Mo). That was a great paper, Dr Sugarbaker. However, I do not understand why you are so light on the radiation. It seems to me that when local control is the issue, you emphasize surgery over radiation. I would have thought that $3000 \mathrm{rad}$ was not quite consistent with the magnitude of the local control you are trying to achieve by that sort of an operation. How did you decide on that particular radiation regimen?

Dr Sugarbaker. We arrived at that dose in a retrograde fashion. We began with $5500 \mathrm{rad}$, the maximal cytologic dose, to the ipsilateral hemithorax. When we combined that with paclitaxel (Taxol) sensitization and the chemotherapy of carboplatin and paclitaxel, we found that a significant number of patients were becoming neutropenic from the amount of marrow that was being radiated. The dose can go as high as 4000 rad, and it really depends on the patient's blood count, but a significant number of patients will become neutropenic from the amount of rib marrow that is being radiated.

Dr L. Penfield Faber (Chicago, Ill). Dr Sugarbaker, this is an outstanding surgical series with a phenomenal mortality rate of $3.8 \%$. Surgeons who have done extrapleural pneumonectomy for mesothelioma fully realize what an achievement this is, as the earlier described mortality rates were much higher. The current mortality rate in our series at the Rush-Presbyterian-St Luke's Medical Center is 7.5\%.

Having done extrapleural pneumonectomies and removed specimens that include the pleura, the pericardium, the lung, and the diaphragm, I find it difficult to understand what constitutes a negative microscopic margin. It can be technically difficult to achieve a negative margin when removing the 
diaphragm from the inferior aspect of the posterior sulcus or at the apex of the chest along the innominate vein and subclavian vessels. You stated that this procedure is really a technique of cytoreduction, and it was implied that there may well be residual microscopic disease. It is difficult for me to comprehend where the microscopic margins are negative and also to understand how a surgical pathologist would evaluate and dissect the specimen to identify negative microscopic margins. Please explain how negative microscopic margins are achieved with this diffuse malignant tumor.

My second question refers to the possible toxic manifestations of paclitaxel coupled with radiation. We have recently completed a pilot phase II study of neoadjuvant therapy for clinically advanced lung cancer using paclitaxel in combination with radiation. We have observed significant complications after surgical resection when pretreating these patients with paclitaxel and radiation. Because of these complications, we no longer use paclitaxel in combination with radiation for neoadjuvant therapy of lung cancer. I would appreciate hearing any comments you might have on the toxic manifestations of paclitaxel in your series.

Dr Sugarbaker. I appreciate your comments. The issue of pathologic resection margins is an important one. The frustration that you outlined-where would you take your sample, how would you approach a specimen-led Dr Joseph Corson (professor of pathology at the Harvard Medical School, and chief of surgical pathology until last year at The Brigham and Women's Hospital) to devise a systematic method some 9 years ago for assessing these specimens; his method involves systematically taking margins from 10 to 15 different points on the specimen, because trying to discern where the specimen looked positive and where it looked negative led to a nonsystematic appraisal.

Dr Corson or his fellow takes the specimen, inks it, and lets us know exactly which of the sections is positive. I would emphasize that in evaluating the survival curves we are looking at a marker of cytoreduction.

I am not suggesting that we are rendering patients diseasefree. However, if you think of 10 to 15 sections and you begin to look at margins negative, margins positive, you are really looking at a quantification of how well your local control procedure accomplished what it set out to do.

The most important lesson is that patients in whom we have achieved near-complete cytoreduction appear to have a survival advantage. This directs us toward local control strategies, such as heated intrathoracic chemotherapy at the time of resection, high-dose radiotherapy, photodynamic therapy, and some of the other areas that would be used against this particular tumor in its most vulnerable and most lethal form, which is local recurrence.

Last, I share your concern regarding paclitaxel radiotherapy. We have found that it is unsuccessful as neoadjuvant therapy in mesothelioma. We have performed extensive surgery after induction therapy and have had a very high complication rate, which led us to abandon that form of treatment. Sometimes patients are referred who have already been treated. Those patients do not do well. Paclitaxel radiotherapy is still an open question. We have witnessed toxicity in conjunction with its use. As Dr Patterson elucidated, we have had to reduce our dose of radiotherapy to avoid toxicity in these patients. 